\title{
Evaluation of a standard format for perinatal counseling
}

\begin{abstract}
Perinatal Counseling provides an opportunity for neonatologists to discuss prognosis and complications of prematurity with expectant mothers. This study evaluates the effectiveness and satisfaction with a standardized consultation format. 42 surveys were completed by 77 eligible mothers who had perinatal consultation between 19 and 34 weeks gestation. 90\% of respondents were satisfied with the amount of influence that they had on the treatment provided \& $66 \%$ were very satisfied with the clarity and amount of information. Our study shows that a standardized format is an effective tool for communicating issues surrounding prematurity, and treatment decisions involved.
\end{abstract}

Keywords: perinatal counseling, prematurity, communication, prognosis, complications
Volume I Issue 7 - 2014

\author{
Eric S Lesser, ${ }^{1,2}$ Jeevith Kumaresan, ' Adel \\ Zauk ${ }^{1,2}$ \\ 'Division of Neonatology, St. Joseph?s Children's Hospital, USA \\ ${ }^{2}$ Division of Neonatology, New York Medical College, USA
}

\begin{abstract}
Correspondence: Eric S Lesser, Division of Neonatology, St Joseph $\square$ s Children $\square$ s Hospital, Suite A24I 8703 Main St, Paterson, New Jersey 07503, USA, Tel (973)-754-2555, Fax (973)754-2567, Email elessermd@yahoo.com
\end{abstract}

Received: October 27, 2014 | Published: November 25, 2014

\section{Background}

Perinatal Counseling allows women at risk for preterm birth to learn about the prognosis of and complications faced by their infant. ${ }^{1,2}$ Perinatal consultation by a neonatologist also establishes some degree of continuity of care for parents of infants who will be in cared for in a complex and intimidating environment. ${ }^{3}$ With 11 neonatologists providing this information our institution initiated a standardized form Figure 1 in 2005 for use during perinatal consults. This two page form contains information on pregnancy complications, gestational age, survival, and expected duration of hospitalization of the infant. Complications of prematurity and long term sequelae are discussed in a systems based checklist to ensure that all applicable topics are covered. Specific information regarding prognosis and outcome is individualized on a case by case basis, depending on gestational age, estimated weight and additional risk factors. Though some of the information presented may be forgotten by parents it is hoped that the consultation will allow parents to feel more comfortable with and engaged in the care of their preterm infants.

\section{Objective}

This study was undertaken to assess the effectiveness of our perinatal consultation and to evaluate maternal satisfaction with the consultation process.

\section{Design/Methods}

After obtaining Institutional Review Board approval and consent from the participants, mothers (aged 18 -35 years, with singleton pregnancies, and no known fetal anomalies) who received prenatal counseling were later interviewed using a structured survey format. Prenatal consults were performed prior to delivery when ordered by an attending obstetrician due to a high likelihood of preterm delivery. Mothers perceptions of the content, tone, and directness of pre delivery counseling and satisfaction with the decision making process were evaluated. Data were for the mothers, infants, and their outcomes. Questionnaires (Figure 2) were completed by the mothers within a week of the consult. Data was analyzed regarding the content and satisfaction of the counseling process.

\section{Results}

Our sample included 42 surveys form 77 eligible mothers.
Respondents were between 19 and 34 weeks gestation (all but one were 23-34 weeks), mean gestational age was 28.6 weeks, with a standard deviation of $+/-3.7$ weeks. All agreed that they were encouraged to ask questions, and that treatment options regarding resuscitation were discussed. $88 \%$ agreed with recommendations for treatment, and $90 \%$ were satisfied with the amount of influence that they had on the treatment provided. $83 \%$ of mothers wanted full neonatal resuscitation, while $16 \%$ preferred treatment only if the infant appeared to be viable. $64 \%$ of respondents indicated that decisions regarding treatment of the infant were made jointly by the parents and neonatologists. $14 \%$ felt that the decision was made by the neonatologists alone. All stated that their opinions were taken into account, but $35 \%$ felt that their opinions were "somewhat" listened to. $95 \%$ were not afraid to ask questions but only $45 \%$ asked questions.

Overall $66 \%$ were very satisfied with the clarity and amount of information. All respondents stated that that probability of survival was discussed. $92 \%$ said that the probability of handicap was discussed, but only $41 \%$ felt that newborn suffering was discussed. All felt that the counselors were very caring and 59\% described them as extremely caring. Lack of follow up was expressed as the only concern. Of the 16 mothers interviewed following delivery, all were satisfied with the treatment their infant received at delivery. Three mothers included in the study gave birth to infants who subsequently died.

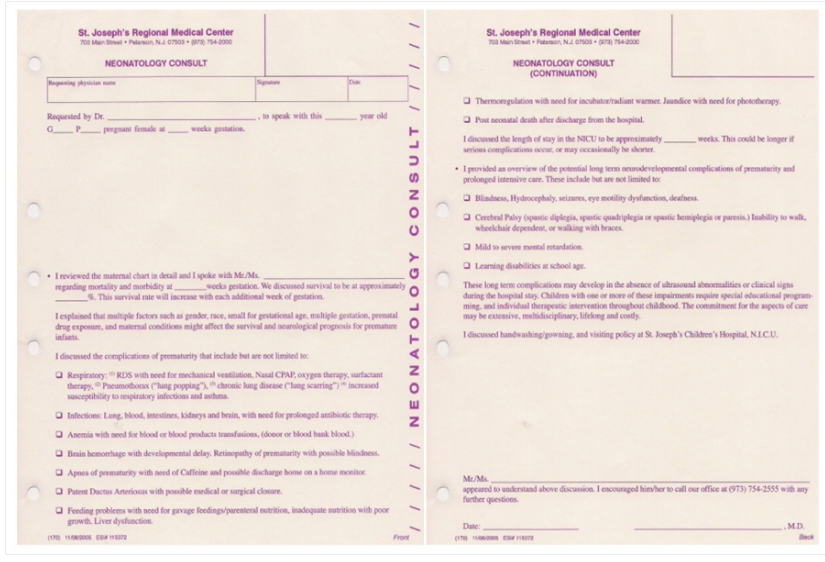

Figure I Consultation Form. 


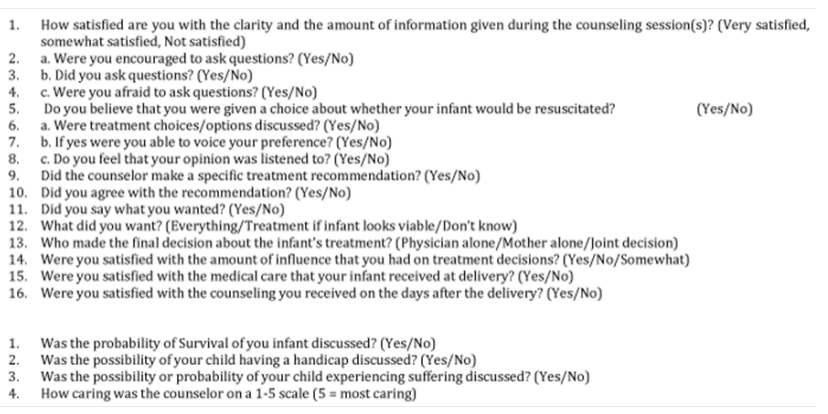

Figure 2 Survey/Questionnaire.

Explanation of long term outcomes and quality of life issues is important especially in infants at the border of viability. ${ }^{4}$ Outcome and Survival data have changed significantly over years., ${ }^{5,6}$ Accuracy of survival data has previously been cited as a problem during prenatal counseling. ${ }^{78}$ Since the time that this study was conducted availability of the NICHD calculator (http://www.nichd.nih.gov/about/org/der/ branches/ppb/programs/epbo/Pages/epbo_case.aspx) has allowed more accurate prediction of outcomes, and that information has now been incorporated into our consultations. The gestational age, estimated birth weight, gender, number (singleton Vs multiple) and use of antenatal steroids (within seven days of delivery) are used to predict outcomes as closely as possible.

\section{Conclusions}

A standardized format is an effective tool for communicating issues surrounding prematurity, and treatment decisions involved. Our results show consistency in the discussion of clinical issues. Follow up to address further questions or concerns may enhance maternal comprehension of these issues. Further feedback from patients may allow the counseling process to be improved upon further enhancing the understanding of the complex issues involved in the birth of a preterm infant.

\section{Acknowledgements}

None.

\section{Conflicts of interests}

None.

Funding

None.

\section{References}

1. Bastek TK, Richardson DK, Zupancic JA, et al. Prenatal Consultation Practices at the Border of Viability: A Regional Survey. Pediatrics. 2005;116(2):407-413.

2. Blanco F, Suresh G, Howard D, et al. Ensuring Accurate Knowledge of Prematurity Outcomes for Prenatal Counseling. Pediatrics. 2005;115(4):e478-e487.

3. Griswold KJ, Fanaroff JM. An Evidence-Based Overview of Prenatal Consultation with a Focus on Infants Born at the Limits of Viability. Pediatrics. 2010;125(4):e931-e937.

4. Halamek LP. The Advantages of Prenatal Consultation by a Neonatologist. J Perinatol. 2001;21(2):116-120.

5. Paul DA, Leef KH, Epps S, et al. Usefulness of the Prenatal Consult: Mother's Response. Pediatric Research. 1999;45:218A.

6. Paul DA, Epps S, Leef KH, et al. Prenatal Consultation with a Neonatologist Prior to Preterm Delivery. J Perinatol. 2001;21(7):431437.

7. Saigal S, Doyle LW. An overview of mortality and sequelae of preterm birth from infancy to adulthood. Lancet. 2008;371(9608):261-269.

8. Wilson-Costello D, Friedman $\mathrm{H}$, Minich $\mathrm{N}$, et al. Improved Neurodevelopmental Outcomes for Extremely Low Birth Weight Infants in 2000-2002. Pediatrics. 2007;119(1):37-45. 